\title{
Flavor changing neutral currents in the asymmetric left-right gauge model
}

\author{
Chia-Feng Chang and Ernest Ma \\ Department of Physics and Astronomy, University of California, \\ Riverside, California 92521, U.S.A. \\ E-mail: chiafeng.chang@email.ucr.edu, ernest.ma@ucr.edu
}

\begin{abstract}
In the $\mathrm{SU}(3)_{C} \times \mathrm{SU}(2)_{L} \times \mathrm{SU}(2)_{R} \times \mathrm{U}(1)_{(B-L) / 2}$ extension of the standard model, a minimal (but asymmetric) scalar sector consists of one $\mathrm{SU}(2)_{R} \times \mathrm{U}(1)_{(B-L) / 2}$ doublet and one $\mathrm{SU}(2)_{L} \times \mathrm{SU}(2)_{R}$ bidoublet. Previous and recent studies have shown that this choice is useful for understanding neutrino mass as well as dark matter. The constraints from flavor changing neutral currents mediated by the scalar sector are discussed in the context of the latest experimental data.
\end{abstract}

KeYwords: Beyond Standard Model, Gauge Symmetry

ARXIV EPRINT: 1806.04812 


\section{Contents}

1 Introduction 1

$\begin{array}{llr}2 & \text { Scalar and gauge sectors } & 2\end{array}$

3 Yukawa sector and the FCNC structure 4

4 Phenomenological constraints $\quad 5$

$4.1 \Delta M_{B_{q}}$ and $\Delta M_{K}$

$4.2 B_{s} \rightarrow \mu^{+} \mu^{-} \quad 7$

4.3 Numerical analysis 8

5 Concluding remarks $\quad 11$

\section{Introduction}

In the conventional left-right extension of the standard model (SM) of quarks and leptons, the gauge symmetry is $\mathrm{SU}(3)_{C} \times \mathrm{SU}(2)_{L} \times \mathrm{SU}(2)_{R} \times \mathrm{U}(1)_{(B-L) / 2}$. The scalar sector must be chosen to break $\mathrm{SU}(2)_{R} \times \mathrm{U}(1)_{(B-L) / 2}$ to $\mathrm{U}(1)_{Y}$ at a scale much higher than that of electroweak symmetry breaking, i.e. $\mathrm{SU}(2)_{L} \times \mathrm{U}(1)_{Y}$ to $\mathrm{U}(1)_{Q}$. This minimum requirement does not uniquely define the scalar particle content, i.e. doublets $\Phi_{L, R}$, triplets $\xi_{L, R}$, and bidoublets $\eta$. There are basically 5 possible choices [1] and they have implications on the nature of neutrino mass, as well as the $\mathrm{SU}(2)_{R}$ breaking scale. The simplest and often neglected choice is to have one $\mathrm{SU}(2)_{R} \times \mathrm{U}(1)_{(B-L) / 2}$ doublet $\Phi_{R}$ and one $\mathrm{SU}(2)_{L} \times \mathrm{SU}(2)_{R}$ bidoublet $\eta$. This implies by itself Dirac neutrino masses, but an inverse seesaw mechanism is easily implemented [2] so that the observed neutrinos are Majorana fermions and the $\mathrm{SU}(2)_{R}$ breaking scale is a few $\mathrm{TeV}$. Whereas flavor-changing neutral-current (FCNC) processes are unavoidable, they are manageable, as shown in ref. [2].

Recently, it has been shown [3] that such a model has another virtue, i.e. the appearance of predestined dark matter. Because of the absence of an $\mathrm{SU}(2)_{L}$ scalar doublet, the insertion of an $\mathrm{SU}(2)_{L}$ fermion triplet $\left(\Sigma^{+}, \Sigma^{0}, \Sigma^{-}\right)$or scalar triplet $\left(\chi^{+}, \chi^{0}, \chi^{-}\right)$automatically guarantees either $\Sigma^{0}$ or $\chi^{0}$ to be stable, so that it is a good candidate for dark matter [4]. Note that $\Sigma^{0}\left(\chi^{0}\right)$ is naturally lighter than $\Sigma^{ \pm}\left(\chi^{ \pm}\right)$from radiative mass splitting [5]. A recently proposed model of $[\mathrm{SU}(2)]^{3}$ dark matter [6] also has this chosen scalar sector.

Since the writing of ref. [2], there are new experimental results on FCNC, mostly in $B$ physics, and new theoretical calculations of their SM contributions. In this paper, we update the resulting phenomenological contraints on this simple scalar sector consisting of only $\Phi_{R}$ and $\eta$. In section 2 the scalar sector is studied as well as the resulting massive gauge 
sector. In section 3 the Yukawa sector is studied and the structure of FCNC couplings to the physical neutral scalars is derived. It is shown that under a simple assumption, all such effects depend only on two scalar masses which are almost degenerate in addition to an unknown unitary $3 \times 3$ matrix $V_{R}$ which is the right-handed analog of the well-known CKM matrix $V_{\mathrm{CKM}}$ for left-handed quarks. In section 4 the experimental data on the $K-\bar{K}$, $B_{d}-\bar{B}_{d}$, and $B_{s}-\bar{B}_{s}$ mass differences, as well as the recent data on $B_{s} \rightarrow \mu^{+} \mu^{-}$, are compared against their SM predictions to constrain the two scalar masses assuming that (A) $V_{R}=V_{\mathrm{CKM}}$ and (B) $V_{R}=1$. In section 5 there are some concluding remarks.

\section{Scalar and gauge sectors}

Under $\mathrm{SU}(3)_{C} \times \mathrm{SU}(2)_{L} \times \mathrm{SU}(2)_{R} \times \mathrm{U}(1)_{B-L}$, we assume one scalar doublet

$$
\Phi_{R}=\left(\begin{array}{c}
\phi_{R}^{+} \\
\phi_{R}^{0}
\end{array}\right) \sim(1,1,2,1 / 2)
$$

and one bidoublet

$$
\eta=\left(\begin{array}{ll}
\eta_{1}^{0} & \eta_{2}^{+} \\
\eta_{1}^{-} & \eta_{2}^{0}
\end{array}\right) \sim(1,2,, 2,0) .
$$

The dual of $\eta$, i.e.

$$
\tilde{\eta}=\sigma_{2} \eta^{*} \sigma_{2}=\left(\begin{array}{cc}
\bar{\eta}_{2}^{0} & -\eta_{1}^{+} \\
-\eta_{2}^{-} & \bar{\eta}_{2}^{0}
\end{array}\right) \sim(1,2,2,0)
$$

is automatically generated and transforms exactly like $\eta$.

The most general Higgs potential consisting of $\Phi_{R}, \eta$, and $\tilde{\eta}$ is given by [2]

$$
\begin{aligned}
V= & m_{R}^{2} \Phi_{R}^{\dagger} \Phi_{R}+m^{2} \operatorname{Tr}\left(\eta^{\dagger} \eta\right)+\frac{1}{2} \mu^{2} \operatorname{Tr}\left(\eta^{\dagger} \tilde{\eta}+\tilde{\eta}^{\dagger} \eta\right)+\frac{1}{2} \lambda_{R}\left(\Phi_{R}^{\dagger} \Phi_{R}\right)^{2}+\frac{1}{2} \lambda_{1}\left[\operatorname{Tr}\left(\eta^{\dagger} \eta\right)\right]^{2} \\
& +\frac{1}{2} \lambda_{2} \operatorname{Tr}\left(\eta^{\dagger} \eta \eta^{\dagger} \eta\right)+\frac{1}{2} \lambda_{3}\left\{\left[\operatorname{Tr}\left(\eta^{\dagger} \tilde{\eta}\right)\right]^{2}+\left[\operatorname{Tr}\left(\tilde{\eta}^{\dagger} \eta\right)\right]^{2}\right\}+\frac{1}{2} \lambda_{4} \operatorname{Tr}\left(\eta^{\dagger} \eta\right)\left[\operatorname{Tr}\left(\eta^{\dagger} \tilde{\eta}+\tilde{\eta}^{\dagger} \eta\right)\right] \\
& +f_{1} \Phi_{R}^{\dagger}\left(\tilde{\eta}^{\dagger} \tilde{\eta}\right) \Phi_{R}+f_{2} \Phi_{R}^{\dagger}\left(\eta^{\dagger} \eta\right) \Phi_{R}+f_{3} \Phi_{R}^{\dagger}\left(\eta^{\dagger} \tilde{\eta}+\tilde{\eta}^{\dagger} \eta\right) \Phi_{R},
\end{aligned}
$$

where all parameters have been chosen real for simplicity. Let $\left\langle\phi_{R}^{0}\right\rangle=v_{R}$ and $\left\langle\eta_{1,2}^{0}\right\rangle=v_{1,2}$, then the minimum of $V$ has a solution where $v_{2} \ll v_{1}$, i.e.

$$
v_{2} \simeq \frac{-\left(\mu^{2}+f_{3} v_{R}^{2}+\lambda_{4} v_{1}^{2}\right) v_{1}}{m^{2}+f_{2} v_{R}^{2}+\left(\lambda_{1}+\lambda_{3}\right) v_{1}^{2}}
$$

with

$$
v_{1}^{2}=\frac{m_{R}^{2} f_{1}-m^{2} \lambda_{R}}{\lambda_{R}\left(\lambda_{1}+\lambda_{2}\right)-f_{1}^{2}}, \quad v_{R}^{2}=\frac{-m_{R}^{2}-f_{1} v_{1}^{2}}{\lambda_{R}} .
$$

In the limit $v_{2}=0$, the physical Higgs bosons are $\phi_{2}^{ \pm}$and $h_{I}=\sqrt{2} \operatorname{Im}\left(\phi_{2}^{0}\right)$ with masses squared

$$
m_{ \pm}^{2}=\left(f_{2}-f_{1}\right) v_{R}^{2}, \quad m_{I}^{2}=\left(f_{2}-f_{1}\right) v_{R}^{2}-\left(\lambda_{2}+\lambda_{3}\right) v_{1}^{2},
$$


and three linear combinations of $h_{1}=\sqrt{2} R e\left(\phi_{1}^{0}\right), h_{2}=\sqrt{2} R e\left(\phi_{2}^{0}\right)$, and $h_{R}=\sqrt{2} \operatorname{Re}\left(\phi_{R}^{0}\right)$, with the $3 \times 3$ mass-squared matrix

$$
\mathcal{M}_{h}^{2}=\left(\begin{array}{ccc}
2\left(\lambda_{1}+\lambda_{2}\right) v_{1}^{2} & 2 \lambda_{4} v_{1}^{2} & 2 f_{1} v_{1} v_{R} \\
2 \lambda_{4} v_{1}^{2} & \left(f_{2}-f_{1}\right) v_{R}^{2}-\left(\lambda_{2}-\lambda_{3}\right) v_{1}^{2} & 2 f_{3} v_{1} v_{R} \\
2 f_{1} v_{1} v_{R} & 2 f_{3} v_{1} v_{R} & 2 \lambda_{R} v_{R}^{2}
\end{array}\right)
$$

Since $v_{1} / v_{R}$ is known to be small, $h_{1,2, R}$ are approximately mass eignestates, with $h_{1}$ almost equal to the observed $125 \mathrm{GeV}$ scalar boson at the Large Hadron Collider (LHC). Note also that $h_{2}$ is almost degenerate with $h_{I}$ in mass. We can make this even more precise by having small $\lambda_{4}$ and $f_{1,3}$.

There are two charged gauge bosons $W_{L}^{ \pm}$and $W_{R}^{ \pm}$in the $2 \times 2$ mass-squared matrix given by

$$
\mathcal{M}_{W}^{2}=\frac{1}{2}\left(\begin{array}{cc}
g_{L}^{2}\left(v_{1}^{2}+v_{2}^{2}\right) & -2 g_{L} g_{R} v_{1} v_{2} \\
-2 g_{L} g_{R} v_{1} v_{2} & g_{R}^{2}\left(v_{R}^{2}+v_{1}^{2}+v_{2}^{2}\right)
\end{array}\right) .
$$

With our assumption that $v_{2} \ll v_{1}, W_{L}-W_{R}$ mixing is negligible. The present LHC bound on the $W_{R}$ mass is $3.7 \mathrm{TeV}[7]$.

There are three neutral gauge bosons, i.e. $W_{3 L}$ from $\mathrm{SU}(2)_{L}, W_{3 R}$ from $\mathrm{SU}(2)_{R}$, and $B$ from $\mathrm{U}(1)_{(B-L) / 2}$, with couplings $g_{L}, g_{R}$, and $g_{B}$ respectively. Let them be rotated to the following three orthonormal states:

$$
\begin{aligned}
A & =\frac{e}{g_{L}} W_{3 L}+\frac{e}{g_{R}} W_{3 R}+\frac{e}{g_{B}} B, \\
Z & =\frac{e}{g_{Y}} W_{3 L}-\frac{e}{g_{L}}\left(\frac{g_{Y}}{g_{R}} W_{3 R}+\frac{g_{Y}}{g_{B}} B\right), \\
Z^{\prime} & =\frac{g_{Y}}{g_{B}} W_{3 R}-\frac{g_{Y}}{g_{R}} B,
\end{aligned}
$$

where

$$
\frac{1}{e^{2}}=\frac{1}{g_{L}^{2}}+\frac{1}{g_{Y}^{2}}, \quad \frac{1}{g_{Y}^{2}}=\frac{1}{g_{R}^{2}}+\frac{1}{g_{B}^{2}} .
$$

The photon $A$ is massless and decouples from $Z$ and $Z^{\prime}$, the latter two forming a masssquared matrix given by

$$
\mathcal{M}_{Z}^{2}=\frac{1}{2}\left(\begin{array}{cc}
\left(g_{L}^{2}+g_{Y}^{2}\right)\left(v_{1}^{2}+v_{2}^{2}\right) & -\left(g_{L} g_{Y}^{2} g_{R} / e g_{B}\right)\left(v_{1}^{2}+v_{2}^{2}\right) \\
-\left(g_{L} g_{Y}^{2} g_{R} / e g_{B}\right)\left(v_{1}^{2}+v_{2}^{2}\right) & \left(g_{R}^{2}+g_{B}^{2}\right) v_{R}^{2}+\left(g_{R}^{2} g_{Y}^{2} / g_{B}^{2}\right)\left(v_{1}^{2}+v_{2}^{2}\right)
\end{array}\right) .
$$

The neutral-current gauge interactions are given by

$$
e A j_{e m}+g_{Z} Z\left(j_{3 L}-\sin ^{2} \theta_{W} j_{e m}\right)+\sqrt{g_{R}^{2}+g_{B}^{2}} Z^{\prime}\left[j_{3 R}+\frac{g_{Y}^{2}}{g_{R}^{2}}\left(j_{3 L}-j_{e m}\right)\right] .
$$

The present LHC bound on the $Z^{\prime}$ mass is $4.1 \mathrm{TeV}$ [8]. The $Z-Z^{\prime}$ mixing is given by $\left(e g_{R} / g_{B} g_{L}\right)\left(m_{Z}^{2} / m_{Z^{\prime}}^{2}\right)$ which is then less than $3.6 \times 10^{-4}$ for $g_{R}=g_{L}$ and within precision measurement bounds. 


\section{Yukawa sector and the FCNC structure}

The fermion content is well-known, i.e.

$$
\begin{array}{ll}
\psi_{L}=\left(\begin{array}{c}
\nu_{e} \\
e
\end{array}\right)_{L} \sim(1,2,1,-1 / 2), \quad \psi_{R}=\left(\begin{array}{c}
\nu_{e} \\
e
\end{array}\right)_{R} \sim(1,1,2,-1 / 2), \\
q_{L}=\left(\begin{array}{l}
u \\
d
\end{array}\right)_{L} \sim(3,2,1,1 / 6), & q_{R}=\left(\begin{array}{l}
u \\
d
\end{array}\right)_{R} \sim(3,1,2,1 / 6),
\end{array}
$$

with the electric charge given by $Q=I_{3 L}+I_{3 R}+(B-L) / 2$. Now the Yukawa couplings between the quarks and the neutral members of the scalar bidoublets are

$$
\left(f_{i j}^{u} \eta_{1}^{0}+f_{i j}^{d} \bar{\eta}_{2}^{0}\right) \bar{u}_{i L} u_{j R}+\left(f_{i j}^{u} \eta_{2}^{0}+f_{i j}^{d} \bar{\eta}_{1}^{0}\right) \bar{d}_{i L} d_{j R}
$$

In the limit $v_{2}=0$, both $u p$ and down quark masses come from only $v_{1}$. Hence

$$
f_{i j}^{u} v_{1}=U_{L}\left(\begin{array}{ccc}
m_{u} & 0 & 0 \\
0 & m_{c} & 0 \\
0 & 0 & m_{t}
\end{array}\right) U_{R}^{\dagger}, \quad f_{i j}^{d} v_{1}=D_{L}\left(\begin{array}{ccc}
m_{d} & 0 & 0 \\
0 & m_{s} & 0 \\
0 & 0 & m_{b}
\end{array}\right) D_{R}^{\dagger},
$$

where $U_{L, R}$ and $D_{L, R}$ are unitary matrices, with

$$
U_{L}^{\dagger} D_{L}=V_{\mathrm{CKM}}, \quad U_{R}^{\dagger} D_{R}=V_{R}
$$

being the known quark mixing matrix for left-handed charged currents and the corresponding unknown one for their right-handed counterpart.

Whereas $Z$ and $Z^{\prime}$ couple diagonally to all quarks, nondiagonal terms appear in the scalar Yukawa couplings. Using eqs. (3.3), (3.4) and (3.5), the FCNC structure is then completely determined, i.e.

$$
\frac{h_{1}}{\sqrt{2} v_{1}}\left(\begin{array}{ccc}
m_{u} & 0 & 0 \\
0 & m_{c} & 0 \\
0 & 0 & m_{t}
\end{array}\right)+\frac{\left(h_{2}-i h_{I}\right)}{\sqrt{2} v_{1}} V_{\mathrm{CKM}}\left(\begin{array}{ccc}
m_{d} & 0 & 0 \\
0 & m_{s} & 0 \\
0 & 0 & m_{b}
\end{array}\right) V_{R}^{\dagger}
$$

for the up quarks, and

$$
\frac{h_{1}}{\sqrt{2} v_{1}}\left(\begin{array}{ccc}
m_{d} & 0 & 0 \\
0 & m_{s} & 0 \\
0 & 0 & m_{b}
\end{array}\right)+\frac{\left(h_{2}+i h_{I}\right)}{\sqrt{2} v_{1}} V_{\mathrm{CKM}}^{\dagger}\left(\begin{array}{ccc}
m_{u} & 0 & 0 \\
0 & m_{c} & 0 \\
0 & 0 & m_{t}
\end{array}\right) V_{R}
$$

for the down quarks. Hence $h_{1}$ behaves as the SM Higgs boson, and at tree-level, all FCNC effects come from $h_{2}$ and $h_{I}$. We may thus use present data to constrain these two masses. Note that all FCNC effects are suppressed by quark masses, so we have an understanding of why they are particularly small in light meson systems.

The analog of eq. (3.3) for leptons is

$$
\left(f_{i j}^{\nu} \eta_{1}^{0}+f_{i j}^{e} \bar{\eta}_{2}^{0}\right) \bar{\nu}_{i L} \nu_{j R}+\left(f_{i j}^{\nu} \eta_{2}^{0}+f_{i j}^{e} \bar{\eta}_{1}^{0}\right) \bar{e}_{i L} e_{j R}
$$


Hence

$$
\left(\mathcal{M}_{\nu}\right)_{i j}=f_{i j}^{\nu} v_{1}+f_{i j}^{e} v_{2}, \quad\left(\mathcal{M}_{e}\right)_{i j}=f_{i j}^{e} v_{1}+f_{i j}^{\nu} v_{2} .
$$

If neutrinos are Dirac fermions, then $\mathcal{M}_{\nu} \simeq 0$ compared to $\mathcal{M}_{e}$, hence $f_{i j}^{\nu}=-\left(v_{2} / v_{1}\right) f_{i j}^{e}$ is a good approximation. The analog of eq. (3.7) for charged leptons is then

$$
\left[\frac{h_{1}}{\sqrt{2} v_{1}}-\frac{\left(h_{2}+i h_{I}\right) v_{2}}{\sqrt{2} v_{1}^{2}}\right]\left(\begin{array}{ccc}
m_{e} & 0 & 0 \\
0 & m_{\mu} & 0 \\
0 & 0 & m_{\tau}
\end{array}\right) \text {. }
$$

\section{Phenomenological constraints}

In the following we consider the contributions of eqs. (3.6), (3.7), and (3.10) to a number of processes sensitive to them in two scenarios: (A) $V_{R}=V_{\mathrm{CKM}}$ and (B) $V_{R}=1$. We compare the most recent experimental data with theoretical SM calculations to obtain constraints coming from the mass differences $\Delta M_{K}, \Delta M_{B_{d}}, \Delta M_{B_{s}}$ of the neutral meson systems of $K-\bar{K}, B_{d}-\bar{B}_{d}, B_{s}-\bar{B}_{s}$ respectively, as well the recent measurement of [9] $B_{s} \rightarrow \mu^{+} \mu^{-}$, i.e.

$$
\overline{\mathcal{B}}\left(B_{s} \rightarrow \mu^{+} \mu^{-}\right)_{\mathrm{LHCb}}=\left(3.0 \pm 0.6_{-0.2}^{+0.3}\right) \times 10^{-9},
$$

with an upper limit $\overline{\mathcal{B}}\left(B_{d} \rightarrow \mu^{+} \mu^{-}\right)_{\text {LHCb }}<3.4 \times 10^{-10}$ at $95 \%$ confidence-level. These values are in agreement with the next-to-leading-order (NLO) electroweak (EW) as well as NNLO QCD predictions [10, 11]:

$$
\overline{\mathcal{B}}\left(B_{s} \rightarrow \mu^{+} \mu^{-}\right)_{\mathrm{SM}}=(3.44 \pm 0.19) \times 10^{-9}, \quad \overline{\mathcal{B}}\left(B_{d} \rightarrow \mu^{+} \mu^{-}\right)_{\mathrm{SM}}=(1.04 \pm 0.09) \times 10^{-10} .
$$

Nevertheless, new physics (NP) contributions are possible within the error bars. In addition, the $K-\bar{K}$ and $B_{q}-\bar{B}_{q}$ mixings, which interfere to obtain time-averaged decay widths [12-14], may also provide possible signals of NP.

The most recently updated SM $\Delta M$ predictions [11, 15-18], and the experimental measurements $[19,20]$ are

$$
\begin{array}{ll}
\Delta M_{K}^{\exp }=(5.296 \pm 0.009) \mathrm{fs}^{-1}, & \Delta M_{K}^{\mathrm{SM}}=(4.73 \pm 1.91) \mathrm{fs}^{-1}, \\
\Delta M_{B_{d}}^{\exp }=(0.5055 \pm 0.0020) \mathrm{ps}^{-1}, & \Delta M_{B_{d}}^{\mathrm{SM}}=(0.642 \pm 0.069) \mathrm{ps}^{-1}, \\
\Delta M_{B_{s}}^{\exp }=(17.757 \pm 0.021) \mathrm{ps}^{-1}, & \Delta M_{B_{s}}^{\mathrm{SM}}=(20.01 \pm 1.25) \mathrm{ps}^{-1} .
\end{array}
$$

Note that $\Delta M_{B_{d}}^{\mathrm{SM}}$ is estimated by the $\mathrm{SU}(3)$-breaking ratio $\xi=1.206(18)(6)$ [11], and the NLO EW, NNLO QCD corrections have been incorporated as well.

\section{$4.1 \quad \Delta M_{B_{q}}$ and $\Delta M_{K}$}

In the SM, other than long-distance contributions [17], $B_{q}-\bar{B}_{q}$ and $K-\bar{K}$ mixings occur mainly via the well-known box diagrams with the exchange of $W^{ \pm}$bosons and the $(u, c, t)$ quarks. In the asymmetric left-right model, the new scalars $h_{2}$ and $h_{I}$ have additional tree-level contributions. We consider the usual operator analysis with Wilson coefficients obtained from the renormalization group (RG). The mass difference between the two mass 
eigenstates of a neutral meson system (see $[19,21]$ for details) may be obtained from the $\Delta F=2$ effective Hamiltonian [22-24]

$$
\mathcal{H}_{\mathrm{eff}}^{\Delta F=2}=\frac{G_{F}^{2}}{16 \pi^{2}} m_{W}^{2}\left(V_{t b} V_{t q}^{*}\right)^{2} \sum_{i} C_{i} \mathcal{O}_{i}+\text { H.c. }
$$

where the operators relevant to the SM and the new scalar contributions are [11]

$$
\begin{aligned}
\mathcal{O}_{\mathrm{SM}} & =\left(\bar{b}^{\alpha} \gamma_{\mu} P_{L} q^{\alpha}\right)\left(\bar{b}^{\beta} \gamma_{\mu} P_{L} q^{\alpha}\right), & \mathcal{O}_{4} & =\left(\bar{b}^{\alpha} P_{L} q^{\alpha}\right)\left(\bar{b}^{\beta} P_{R} q^{\beta}\right), \\
\mathcal{O}_{2} & =\left(\bar{b}^{\alpha} P_{L} q^{\alpha}\right)\left(\bar{b}^{\beta} P_{L} q^{\beta}\right), & \tilde{\mathcal{O}}_{2} & =\left(\bar{b}^{\alpha} P_{R} q^{\alpha}\right)\left(\bar{b}^{\beta} P_{R} q^{\beta}\right), \\
\mathcal{O}_{3} & =\left(\bar{b}^{\alpha} P_{L} q^{\beta}\right)\left(\bar{b}^{\beta} P_{L} q^{\alpha}\right), & & \tilde{\mathcal{O}}_{3}=\left(\bar{b}^{\alpha} P_{R} q^{\beta}\right)\left(\bar{b}^{\beta} P_{R} q^{\alpha}\right),
\end{aligned}
$$

for the $B_{d}-\bar{B}_{d}$ and $B_{s}-\bar{B}_{s}$ systems. In the case of $K-\bar{K}$, we just change $b$ to $s$ and $q$ to $d$ in the above. $P_{R}$ and $P_{L}$ are right- and left-handed projection operators $\left(1 \pm \gamma_{5}\right) / 2$, respectively. $\alpha$ and $\beta$ are color indices. We follow the details in [22] with recent updates $[11,25]$ for $B_{q}$ as well as [17] for $K$. After ignoring terms that are suppressed by light quark masses, we obtain

$$
C_{\mathrm{SM}}^{q}=4 S_{0}\left(x_{t}\right) \eta_{2 B}(\mu), \quad C_{\mathrm{SM}}^{K}=4 \lambda_{c}^{2} \eta_{c c} S_{0}\left(x_{c}\right) / \lambda_{t}^{2}+4 \eta_{t t} S_{0}\left(x_{t}\right)+8 \lambda_{c} \eta_{c t} S_{0}\left(x_{c}, x_{t}\right) / \lambda_{t}
$$

with $\lambda_{x} \equiv V_{x s} V_{x d}^{*}$. The Inami-Lim function $S_{0}\left(x_{i}, x_{j}\right)$ with $x_{q} \equiv\left(m_{q}\left(m_{q}\right) / m_{W}\right)^{2}$ describes the electroweak corrections in one loop [26]. The factors $\eta_{i}$ are perturbative QCD corrections at NLO [22], as well as $[27]([23])$ for the new $B_{q}(K)$ terms. Since the QCD corrections generate nondiagonal entries, the color mixed operators should be considered as well at low scale $[28]$ (see also $[15,29,30])$.

Noting that $\left\langle\mathcal{O}_{2,3}\right\rangle=\left\langle\tilde{\mathcal{O}}_{2,3}\right\rangle$ in QCD, we consider the relevant operators for $B_{q}-\bar{B}_{q}$ mixing in terms of their bag parameters [11,31],

$$
\left\langle\mathcal{O}_{1}^{q}\right\rangle(\mu)=c_{1} f_{B_{q}}^{2} M_{B_{q}}^{2} B_{B_{q}}^{(1)}(\mu)
$$

and

$$
\left\langle\mathcal{O}_{i}^{q}\right\rangle(\mu)=c_{i}\left(\frac{M_{B_{q}}}{m_{b}(\mu)+m_{q}}\right)^{2} f_{B_{q}}^{2} M_{B_{q}}^{2} B_{B_{q}}^{(i)}(\mu), \quad i=2,3
$$

and

$$
\left\langle\mathcal{O}_{i}^{q}\right\rangle(\mu)=c_{i}\left[\left(\frac{M_{B_{q}}}{m_{b}(\mu)+m_{q}}\right)^{2}+d_{i}\right] f_{B_{q}}^{2} M_{B_{q}}^{2} B_{B_{q}}^{(i)}(\mu), \quad i=4,5,
$$

with $c_{i}=\{2 / 3,-5 / 12,1 / 12,1 / 2,1 / 6\}, d_{4}=1 / 6$, and $d_{5}=3 / 2$. The decay constants and bag parameters $B_{B_{q}}^{(i)}$ include all nonperturbative effects. The lattice calculation has been done in [11] for $B_{q}$ with in the scheme of [29], as well as [16] for $K$. The renormalization group evolution effects are considered in $[23,27]$. 
In the asymmetric left-right model, the tree-level $h_{2}$ and $h_{I}$ contributions to the Wilson coefficients at the new physics scale $\mu_{\mathrm{NP}}$ are

$$
\begin{aligned}
C_{2} & =-\frac{1}{2} \kappa\left[\left(V_{d}^{\dagger}\right)_{b, q}\right]^{2}\left(\frac{1}{m_{2}^{2}}-\frac{1}{m_{I}^{2}}\right), \quad \tilde{C}_{2}=-\frac{1}{2} \kappa\left[\left(V_{d}\right)_{b, q}\right]^{2}\left(\frac{1}{m_{2}^{2}}-\frac{1}{m_{I}^{2}}\right), \\
C_{4} & =-\kappa\left(V_{d}\right)_{b, q}\left(V_{d}^{\dagger}\right)_{b, q}\left(\frac{1}{m_{2}^{2}}+\frac{1}{m_{I}^{2}}\right),
\end{aligned}
$$

where $\kappa=16 \pi^{2} / G_{F}^{2} m_{W}^{2}\left(V_{t b} V_{t q}^{*}\right)^{2}$, and the matrix $V_{d}$ comes from the second term of eq. (3.7). The $B_{q}$ mass difference is thus given by

$$
\begin{aligned}
2 M_{12}^{q}= & \frac{\left\langle\bar{B}_{q}\left|\mathcal{H}_{\mathrm{eff}}^{\Delta F=2}\right| B_{q}\right\rangle}{M_{B_{q}}}=\frac{G_{F}^{2}}{16 \pi^{2}} \frac{m_{W}^{2}}{M_{B_{q}}}\left(V_{t b} V_{t q}^{*}\right)^{2} \\
& \times\left[C_{\mathrm{SM}}^{q} c_{1} f_{B_{q}}^{2} M_{B_{q}}^{2} \hat{B}_{B_{q}}^{(1)}+\left(C_{2}+\tilde{C}_{2}\right)\left(\eta_{22}\left\langle\mathcal{O}_{2}^{q}\right\rangle+\eta_{32}\left\langle\mathcal{O}_{3}^{q}\right\rangle\right)+C_{4} \eta_{4}\left\langle\mathcal{O}_{4}^{q}\right\rangle\right],
\end{aligned}
$$

where $\eta_{4} \simeq 3.90, \eta_{22} \simeq 2.25$ and $\eta_{32} \simeq-0.12$, [27, 32]. Similarly, the $K^{0}$ mass difference is

$$
2 M_{12}^{K}=\frac{G_{F}^{2} m_{W}^{2}}{16 \pi^{2}} f_{K}^{2} M_{K}\left(V_{t s} V_{t d}^{*}\right)^{2}\left[C_{\mathrm{SM}}^{K} P_{1}^{V L L}+C_{2} P_{1}^{S L L}+C_{4} P_{2}^{L R}\right],
$$

where $P_{1,2}$ are given in $[17,23]$ and a recently updated lattice simulation [16]. Hence

$$
\Delta M_{K}=2 \operatorname{Re}\left[M_{12}^{K}\right], \quad \Delta M_{B_{q}}=2\left|M_{12}^{q}\right|, \quad \text { and } \quad \phi_{q}^{M}=\arg M_{12}^{q} .
$$

Note that $\phi_{s}^{M}$ may deviate [14] from the SM value, i.e. $\phi_{s}^{M}=\phi_{s}^{\mathrm{SM}}+\phi_{s}^{N P}$. A nonzero $\phi_{s}^{N P}$ would contribute to the $C P$ violation effect in the $B_{s} \rightarrow(J / \psi) \phi$ decay (see [33] and the recent review [21]). Present data imply the constraint $\phi_{s}^{N P}=0.4^{\circ} \pm 1.9^{\circ}$ [34]. For $B_{d}$, the phase constraint is $\phi_{d}^{N P}=-3.8^{\circ} \pm 4.4^{\circ}[35,36]$.

\section{$4.2 \quad B_{s} \rightarrow \mu^{+} \mu^{-}$}

The scalars $h_{2}$ and $h_{I}$ contribute not only to the mass difference of $B_{s}$, but also to the decay of $B_{s} \rightarrow \mu^{+} \mu^{-}$at tree level. The SM contribution is dominated by the operator $\mathcal{O}_{10}^{S M}$, so we ignore other possible SM operators [24, 25]. The effective Hamiltonian is given by $[10,37]$

$$
\mathcal{H}_{\mathrm{eff}}=-\frac{G_{F}}{\sqrt{2}} \frac{\alpha_{e m}}{\pi s_{W}^{2}} V_{t b} V_{t s}^{*}\left(C_{10}^{\mathrm{SM}} \mathcal{O}_{10}^{\mathrm{SM}}+C_{S} \mathcal{O}_{S}+C_{P} \mathcal{O}_{P}+C_{S}^{\prime} \mathcal{O}_{S}^{\prime}+C_{P}^{\prime} \mathcal{O}_{P}^{\prime}\right)+\text { H.c. }
$$

where $\alpha_{e m}$ is the fine structure constant, and $s_{W}^{2} \equiv \sin ^{2} \theta_{W}$ with $\theta_{W}$ the weak mixing angle. The operators are defined as

$$
\begin{aligned}
& \mathcal{O}_{10}^{\mathrm{SM}}=\left(\bar{q} \gamma^{\mu} P_{L} b\right)\left(\bar{\mu} \gamma^{\mu} \gamma_{5} \mu\right), \quad \mathcal{O}_{P}=m_{b}\left(\bar{q} P_{R} b\right)\left(\bar{\mu} \gamma_{5} \mu\right), \quad \mathcal{O}_{P}^{\prime}=m_{b}\left(\bar{q} P_{L} b\right)\left(\bar{\mu} \gamma_{5} \mu\right), \\
& \mathcal{O}_{S}=m_{b}\left(\bar{q} P_{R} b\right)(\bar{\mu} \mu), \quad \mathcal{O}_{S}^{\prime}=m_{b}\left(\bar{q} P_{L} b\right)(\bar{\mu} \mu) .
\end{aligned}
$$

Including the $b$ quark mass $m_{b}$ makes those operators as well as their Wilson coefficients to be renormalization-group invariant [25]. For the NLO SM contribution, we use a numerical value approximated by [25]

$$
C_{10}^{\mathrm{SM}}=-0.9380\left(\frac{m_{t}^{p}}{173.1 \mathrm{GeV}}\right)^{1.53}\left(\frac{\alpha_{s}\left(m_{Z}\right)}{0.1184}\right)^{-0.09},
$$


where $m_{t}^{p}$ is the $t$ quark pole mass. The contributions of NLO EW and NNLO QCD have been computed by [38-42]. The non-SM Wilson coefficients are given by tree-level $h_{2}$ or $h_{I}$ exchange, i.e.

$$
\begin{aligned}
& C_{P}=\tilde{\kappa}\left(V_{d}\right)_{s, b}\left(i \frac{\operatorname{Im}\left(V_{p}\right)_{\mu \mu}}{m_{2}^{2}}-\frac{\operatorname{Re}\left(V_{p}\right)_{\mu \mu}}{m_{I}^{2}}\right), \quad C_{P}^{\prime}=\tilde{\kappa}\left(V_{d}^{\dagger}\right)_{s, b}\left(i \frac{\operatorname{Im}\left(V_{p}\right)_{\mu \mu}}{m_{2}^{2}}+\frac{\operatorname{Re}\left(V_{p}\right)_{\mu \mu}}{m_{I}^{2}}\right), \\
& C_{S}=\tilde{\kappa}\left(V_{d}\right)_{s, b}\left(\frac{\operatorname{Re}\left(V_{p}\right)_{\mu \mu}}{m_{2}^{2}}-i \frac{\operatorname{Im}\left(V_{p}\right)_{\mu \mu}}{m_{I}^{2}}\right), \quad C_{S}^{\prime}=\tilde{\kappa}\left(V_{d}^{\dagger}\right)_{s, b}\left(\frac{\operatorname{Re}\left(V_{p}\right)_{\mu \mu}}{m_{2}^{2}}+i \frac{\operatorname{Im}\left(V_{p}\right)_{\mu \mu}}{m_{I}^{2}}\right),
\end{aligned}
$$

where $\tilde{\kappa}=\pi^{2} / G_{F}^{2} m_{b} m_{W}^{2} V_{t b} V_{t s}^{*}$, and the matrix $V_{p}$ comes from the second term of eq. (3.10). The form factors are

$$
\left\langle 0\left|\bar{q} \gamma_{\mu} \gamma_{5} b\right| \bar{B}_{q}(p)\right\rangle=i f_{B_{q}} p_{\mu}, \quad\left(m_{b}\right)\left\langle 0\left|\bar{q} \gamma_{5} b\right| \bar{B}_{q}(p)\right\rangle=-i f_{B_{q}} \frac{M_{B_{q}}^{2}}{m_{b}+m_{q}} m_{b} .
$$

From the above, the branching fraction of $B_{s} \rightarrow \mu^{+} \mu^{-}$is then [12]

$$
\mathcal{B}\left(B_{q} \rightarrow \mu^{+} \mu^{-}\right)=\frac{\tau_{B_{q}} G_{F}^{4} m_{W}^{4}}{8 \pi^{5}}\left|V_{t b} V_{t s}^{*}\right|^{2} f_{B}^{2} m_{\mu}^{2} m_{B} \sqrt{1-\frac{4 m_{\mu}^{2}}{m_{B}^{2}}}\left(|P|^{2}+|S|^{2}\right),
$$

where $m_{B_{s}}, \tau_{B_{s}}$ and $f_{B_{s}}$ denote the mass, lifetime and decay constant of the $B_{s}$ meson, respectively. The amplitudes $P$ and $S$ are defined as [14]

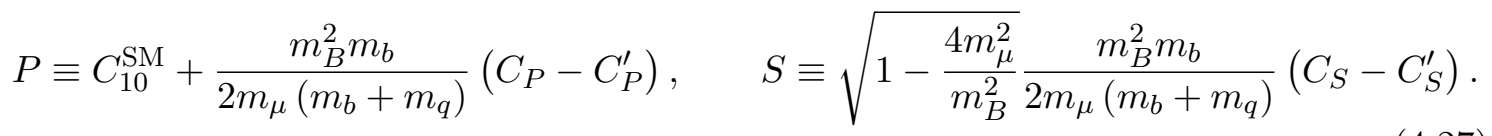

To compare against experimental data, the time-integrated branching fraction is discussed extensively in $[12-14,43]$, i.e.

$$
\overline{\mathcal{B}}\left(B_{s} \rightarrow \mu^{+} \mu^{-}\right)_{\exp }=\left(\frac{1+\mathcal{A}_{\Delta \Gamma} y_{s}}{1-y_{s}^{2}}\right) \mathcal{B}\left(B_{s} \rightarrow \mu^{+} \mu^{-}\right),
$$

where $y_{s}=\Delta \Gamma_{s} / 2 \Gamma_{s}\left(\Gamma_{s}\right.$ being the average $B_{s}$ decay width) and [33]

$$
\mathcal{A}_{\Delta \Gamma}=\frac{|P|^{2} \cos \left(2 \phi_{P}-\phi_{s}^{N P}\right)-|S|^{2} \cos \left(2 \phi_{S}-\phi_{s}^{N P}\right)}{|P|^{2}+|S|^{2}},
$$

with

$$
S=|S| e^{i \phi_{S}}, \quad P=|P| e^{i \phi_{P}}, \quad 2 \arg \left(V_{t s} V_{t b}^{*}\right) \equiv \phi_{s}^{\mathrm{SM}} .
$$

\subsection{Numerical analysis}

We now discuss the experimental constraints on the two scalar masses $m_{2}$ and $m_{I}$. We allow for the theoretical uncertainties in computing $\Delta M_{K}, \Delta M_{B_{q}}$ and $B_{s} \rightarrow \mu^{+} \mu^{-}$which arise mainly from the decay constant $f_{B_{q}}$ (and the bag parameters $\hat{B}_{q}^{(i)}$ ) and the combination of 


\begin{tabular}{|c|c|c|c|c|c|}
\hline Parameter & Value & Ref. & Parameter & Value & Ref. \\
\hline$m_{W}$ & $80.385(15) \mathrm{GeV}$ & {$[19]$} & $m_{t}^{p}$ & $173.21(87) \mathrm{GeV}$ & {$[19]$} \\
\hline$G_{F}$ & $1.1663787(6) \times 10^{-5} \mathrm{GeV}^{-2}$ & {$[19]$} & $m_{t}\left(m_{t}\right)$ & $162.5(11) \mathrm{GeV}$ & {$[44]$} \\
\hline$\hbar$ & $6.582119514(40) \times 10^{-25} \mathrm{GeV} \mathrm{s}$ & {$[19]$} & $m_{b}\left(m_{b}\right)$ & $4.19(18) \mathrm{GeV}$ & {$[44]$} \\
\hline$\tau_{B_{s}}$ & $1.510(5) \mathrm{ps}$ & {$[19]$} & $m_{c}\left(m_{b}\right)$ & $0.934_{-0.120}^{+0.058} \mathrm{GeV}$ & {$[44]$} \\
\hline$\Delta \Gamma_{s}$ & $0.082(7) \mathrm{ps}^{-1}$ & {$[19]$} & $m_{s}\left(m_{b}\right)$ & $84_{-17}^{+26} \mathrm{MeV}$ & {$[44]$} \\
\hline$M_{B_{s}}$ & $5.36689(19) \mathrm{GeV}$ & {$[19]$} & $m_{u}\left(m_{b}\right)$ & $2.02(60) \mathrm{MeV}$ & {$[44]$} \\
\hline$M_{B_{d}}$ & $5.27961(16) \mathrm{GeV}$ & {$[19]$} & $m_{d}\left(m_{b}\right)$ & $4.12(69) \mathrm{MeV}$ & {$[44]$} \\
\hline$M_{K}$ & $0.497611(13) \mathrm{GeV}$ & {$[19]$} & $m_{c}\left(m_{c}\right)$ & $1.29_{-0.11}^{+0.05} \mathrm{GeV}$ & {$[44]$} \\
\hline$\alpha_{s}^{(5)}\left(m_{Z}\right)$ & $0.1181(11)$ & {$[19]$} & $V_{u s}$ & $0.22508_{-0.00028}^{+0.0030}$ & {$[35]$} \\
\hline$f_{B_{s}}$ & $227.2(34) \mathrm{MeV}$ & {$[11]$} & $V_{c b}$ & $0.04181_{-0.00060}^{+0.00028}$ & {$[35]$} \\
\hline$\gamma_{\mathrm{CKM}}$ & $1.141_{-0.020}^{+0.017}$ & {$[35]$} & $\left|V_{u b} / V_{c b}\right|$ & $0.0889(14)$ & {$[35]$} \\
\hline$f_{K}$ & $0.1562(9) \mathrm{GeV}$ & {$[15]$} & $\hat{B}_{K}$ & $0.7625(97)$ & {$[15]$} \\
\hline$B_{K}^{(2)}(2 \mathrm{GeV})$ & $0.568(26)$ & {$[16]$} & $B_{K}^{(3)}(2 \mathrm{GeV})$ & $0.382(21)$ & {$[16]$} \\
\hline$B_{K}^{(4)}(2 \mathrm{GeV})$ & $0.984(67)$ & {$[16]$} & $B_{K}^{(5)}(2 \mathrm{GeV})$ & $0.714(78)$ & {$[16]$} \\
\hline$f_{B_{d}}$ & $190.9(4.1) \mathrm{MeV}$ & {$[11]$} & $\eta_{c c}$ & $1.87(76)$ & {$[45]$} \\
\hline$\Lambda_{\mathrm{QCD}}^{(5)}$ & $0.226 \mathrm{GeV}$ & {$[22]$} & $\eta_{c t}$ & $0.496(47)$ & {$[45]$} \\
\hline$f_{B_{s}} \sqrt{\hat{B}_{B_{s}}^{(1)}}$ & $274.6 \pm 11.1 \mathrm{MeV}$ & {$[11]$} & $\eta_{t t}$ & $0.5765(65)$ & {$[45]$} \\
\hline$f_{B_{d}} \sqrt{\hat{B}_{B_{d}}^{(1)}}$ & $227.7 \pm 11.8 \mathrm{MeV}$ & {$[11]$} & $\eta_{2 B}$ & $0.55210(62)$ & {$[37]$} \\
\hline
\end{tabular}

Table 1. List of input parameters (including table XIII of [11] in the scheme of [29]).

CKM matrix elements $\left|V_{t s}^{*} V_{t b}\right|$ (i.e. $\left|V_{c b}\right|$ as well as $\left|V_{u b}\right|$, from the unitarity of $V_{\mathrm{CKM}}$ ) [11]. We note that there is a long-standing discrepancy between the determinations of $V_{u b}$ from inclusive and exclusive $B$ decays. We adopt the recent averaged CKM matrix elements by the CKMfitter group [35], and use running quark masses [44]. Our input parameters are given in table 1 , and the scales used are $\left\{\mu_{K}, \mu_{b}, \mu_{\mathrm{NP}}\right\}=\{2,3,1000\} \mathrm{GeV}$.

Flavor-changing neutral scalar couplings to quarks are studied in two scenarios, where the $\mathrm{SU}(2)_{R}$ charged-current mixing matrix $V_{R}$ in eq. (3.5) is given either by the CKM matrix (Scenario A), i.e. $V_{R} \equiv V_{\mathrm{CKM}}$, or just the identity matrix (Scenario B), i.e. $V_{R} \equiv 1$. Tree-level contributions exist from the exchange of the new CP-even scalar $h_{2}$ or the CPodd scalar $h_{I}$, as shown in figure 1. The Wilson coefficients for $\Delta M_{B_{q}}$ and $\Delta M_{K}$ are given in eqs. (4.14) and (4.15). The $B_{s} \rightarrow \mu^{+} \mu^{-}$contribution comes from eqs. (4.22) and (4.23).

In Scenario A, since the mixing matrix $V_{d}$ is Hermitian [see eq. (3.7)], fine-tuned cancellations between $C_{2}, \tilde{C}_{2}$ and $C_{4}$ appear only if a large ratio $\left(m_{2}^{2}-m_{I}^{2}\right) /\left(m_{2}^{2}+m_{I}^{2}\right)$ appears, [see eq. (4.16)], but this cannot happen within the given parameter space. Therefore, the $\Delta M_{B_{q}}$ constraints only allow the (red, black) area without fine-tuning, i.e. $m_{2}$ and/or $m_{I} \geq 13.5 \mathrm{TeV}$. On the other hand, the $h_{2}-h_{I}$ mass-squared difference $m_{2}^{2}-m_{I}^{2}=2 \lambda_{3} v_{1}^{2}$ restricts it to only a thin line in the region of heavier masses, i.e. $m_{2} \simeq m_{I}$. Their overlap 


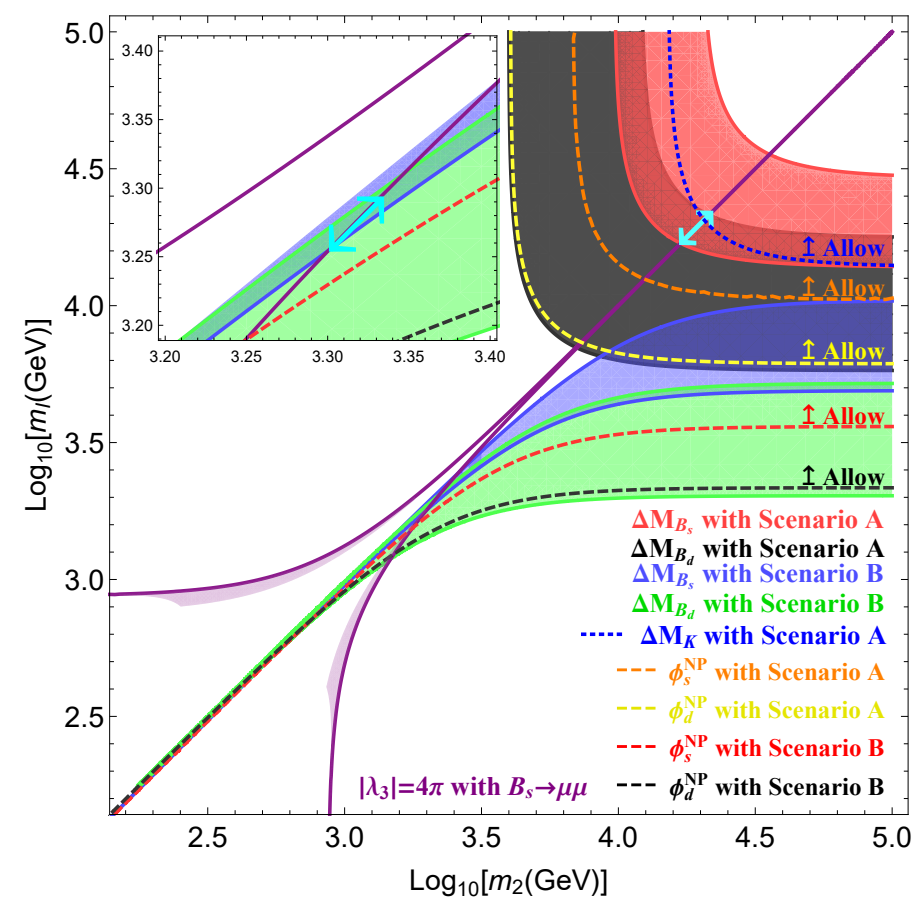

Figure 1. Allowed parameter space in the $\left(m_{2}, m_{I}\right)$ plane. The (red, black) and (blue, green) shaded regions correspond to Scenario $\mathrm{A}\left(V_{R}=V_{\mathrm{CKM}}\right)$ and Scenario B $\left(V_{R}=1\right)$ within the $1 \sigma$ region of $\Delta M_{B_{q}}$, respectively. The purple shaded regions correspond to Scenario (A, B) with $v_{2}<\frac{1}{2} v_{1}$ from the constraint $m_{2}^{2}-m_{I}^{2}=2 \lambda_{3} v_{1}^{2}$ with $\left|\lambda_{3}\right|=4 \pi$ and its overlap within the $1 \sigma$ region of $\overline{\mathcal{B}}\left(B_{s} \rightarrow \mu^{+} \mu^{-}\right)$. The dotted blue line corresponds to the $\Delta M_{K}$ constraint, including the LD effects. The light-orange(dashed yellow line) is shown at the $1 \sigma$ experimental CP phase constraint of the $B_{s}\left(B_{d}\right)$ phase in Scenario A, and the dashed red(black) line is the $B_{s}\left(B_{d}\right)$ phase constraint in Scenario B, which excludes the lower-right region of this figure. The dark-purple lines show the $v_{2} \rightarrow 0$ limit, i.e. a null contribution to $B_{s} \rightarrow \mu \mu$ from new physics. The survival parameter spaces under $\Delta M_{s, d}$ are marked by cyan $\uparrow$. The input parameters are from table 1 .

shows a strong constraint indicated by an arrow (cyan) in figure 1. If the $\Delta M_{K}$ constraint is included, then this tiny allowed region is ruled out if only the short-distance (SD) contribution is considered. Adding the long-distance (LD) contributions from $\pi$ and $\eta^{\prime}$ exchange [46, 47]

$$
\Delta m_{K}=\Delta m_{K}^{\mathrm{SD}}+\left.\Delta m_{K}^{\mathrm{LD}}\right|_{\pi \pi}+\left.\Delta m_{K}^{\mathrm{LD}}\right|_{\eta^{\prime}}
$$

with

$$
\left.\Delta m_{K}^{\mathrm{LD}}\right|_{\pi \pi}=0.4 \Delta m_{K}^{\mathrm{exp}},\left.\quad \Delta m_{K}^{\mathrm{LD}}\right|_{\eta^{\prime}}=-0.3 \Delta m_{K}^{\mathrm{exp}},
$$

a consistent overlap with the data may be obtained. Although the LD contributions are still not well understood, with somewhat large uncertainties [17], these terms shift the SM contribution and allow Scenario A to survive. In summary, the above constraints with LD physics allow the masses to lie within the region $20.0 \mathrm{TeV} \leq m_{2} \simeq m_{I} \leq 22.8 \mathrm{TeV}$.

In Scenario B, the asymmetric mixing matrix elements e.g. $\left(V_{d}\right)_{b, s} \simeq-0.01\left(V_{d}^{\dagger}\right)_{b, s}$ result in cancellations between Wilson coefficients $C_{2}, \tilde{C}_{2}$ and $C_{4}$ if $\left(m_{2}^{2}-m_{I}^{2}\right) /\left(m_{2}^{2}+m_{I}^{2}\right) \simeq 0.01$. Hence lighter $m_{2}, m_{I}$ masses from $\Delta M_{B_{q}}$ are not ruled out in the (blue, green) area of 
figure 1 where $\left|\lambda_{3}\right|=4 \pi$ has been used. The two branches (purple) represent the model restrictions on $\left(m_{2}, m_{I}\right)$ depending on the sign of $\lambda_{3}$. If a value of $\left|\lambda_{3}\right|$ less than $4 \pi$ is used, then the region between these two branches will be filled in. Since our model contribution to $B_{s} \rightarrow \mu^{+} \mu^{-}$is proportional to $v_{2}$ which is always assumed to be small so far, there is no constraint from it unless $v_{2}$ is sizeable. For $\left|\lambda_{3}\right|=4 \pi$, if we also assume $v_{2}<0.5 v_{1}$, then within $1 \sigma$ of the $B_{s} \rightarrow \mu^{+} \mu^{-}$experimental rate, the allowed region cuts off for small $\left(m_{2}, m_{I}\right)$, as shown (purple) in figure 1 . The allowed region with $\lambda_{3}=4 \pi$ in Scenario B is indicated by an arrow (cyan) in the subgraph, i.e. $1.80 \leq m_{I} \leq 2.45 \mathrm{TeV}$. For $\lambda_{3}<4 \pi$, a thin region opens up above the purple line. As for $\Delta M_{K}$ in Scenario $\mathrm{B}$, this result is not affected whether LD contributions are included or not.

From eq. (3.6), we see that $D^{0}-\bar{D}^{0}$ mixing is suppressed by down-quark masses in the asymmetric left-right model. It does not provide a tighter constraint [32, 48, 49].

\section{Concluding remarks}

We have studied the possible contributions of the heavy scalars $h_{2}$ and $h_{I}$ in the asymmetric left-right model to $B_{q}-\bar{B}_{q}$ mixings as well as $B_{s} \rightarrow \mu^{+} \mu^{-}$. We find that improvements of the fit to experimental data within $1 \sigma$ are possible, as shown in figure 1 . In the scenario with the right-handed charged-current mixing matrix $V_{R}$ equal to $V_{\mathrm{CKM}}$, we predict $m_{2} \simeq m_{I}$ to be between 20.0 and $22.8 \mathrm{TeV}$. If $V_{R}=1$, then $m_{I} \simeq 1.80$ to $2.45 \mathrm{TeV}$, and $m_{2} \simeq 2.00$ to $2.60 \mathrm{TeV}$ for $\lambda_{3}=4 \pi$ and small $v_{2}$.

If the doublet $\Phi_{R}$ is replaced with the triplet $\left(\xi_{R}^{++}, \xi_{R}^{+}, \xi_{R}^{0}\right)$, the FCNC analysis remains the same. What will change is that $\nu_{R}$ will acquire a large Majorana mass and the usual neutrinos will get seesaw Majorana masses. A doubly-charged physical scalar $\xi_{R}^{ \pm \pm}$will also appear and decays to $e^{ \pm} e^{ \pm}$. In addition, there are more candidates for predestined dark matter [3], i.e. scalar $\mathrm{SU}(2)_{L}$ triplet, fermion singlet, fermion bidoublet, fermion $\mathrm{SU}(2)_{L}$ triplet, and fermion $\mathrm{SU}(2)_{R}$ triplet.

The new physics contributions of $h_{2}$ and $h_{I}$ are summarized in eqs. (3.6), (3.7), and (3.10). Whereas lepton flavor universality (LFU) is violated, the effect is small, being suppressed by lepton masses. Hence this model is unable to explain recent $B$ decay anomalies [50] observed at the LHC. Other new physics effects outside the scope of this model would be required.

\section{Acknowledgments}

This work was supported in part by the U.S. Department of Energy Grant No. DESC0008541.

Open Access. This article is distributed under the terms of the Creative Commons Attribution License (CC-BY 4.0), which permits any use, distribution and reproduction in any medium, provided the original author(s) and source are credited. 


\section{References}

[1] E. Ma, Neutrino mass and the $\mathrm{SU}(2)_{R}$ breaking scale, Phys. Rev. D 69 (2004) 011301 [hep-ph/0308092] [INSPIRE].

[2] A. Aranda, J.L. Diaz-Cruz, E. Ma, R. Noriega and J. Wudka, Asymmetric Higgs Sector and Neutrino Mass in an $\mathrm{SU}(2)_{R}$ Model, Phys. Rev. D 80 (2009) 115003 [arXiv:0909.1754] [INSPIRE].

[3] E. Ma, Predestined Dark Matter in Gauge Extensions of the Standard Model, LHEP 1 (2018) 1 [arXiv:1803.03891] [INSPIRE].

[4] E. Ma and D. Suematsu, Fermion Triplet Dark Matter and Radiative Neutrino Mass, Mod. Phys. Lett. A 24 (2009) 583 [arXiv:0809.0942] [InSPIRE].

[5] M. Sher, Charged leptons with nanosecond lifetimes, Phys. Rev. D 52 (1995) 3136 [hep-ph/9504257] [INSPIRE].

[6] E. Ma, [SU(2)] ${ }^{3}$ dark matter, Phys. Lett. B 780 (2018) 533 [arXiv:1712.08994] [INSPIRE].

[7] ATLAS collaboration, Search for High-Mass Resonances Decaying to $\tau \nu$ in pp Collisions at $\sqrt{s}=13$ TeV with the ATLAS Detector, Phys. Rev. Lett. 120 (2018) 161802 [arXiv: 1801.06992] [INSPIRE].

[8] ATLAS collaboration, Search for new high-mass phenomena in the dilepton final state using $36 \mathrm{fb}^{-1}$ of proton-proton collision data at $\sqrt{s}=13 \mathrm{TeV}$ with the ATLAS detector, JHEP 10 (2017) 182 [arXiv:1707.02424] [INSPIRE].

[9] LHCb collaboration, Measurement of the $B_{s}^{0} \rightarrow \mu^{+} \mu^{-}$branching fraction and effective lifetime and search for $B^{0} \rightarrow \mu^{+} \mu^{-}$decays, Phys. Rev. Lett. 118 (2017) 191801 [arXiv: 1703.05747] [INSPIRE].

[10] C. Bobeth, M. Gorbahn, T. Hermann, M. Misiak, E. Stamou and M. Steinhauser, $B_{s, d} \rightarrow l^{+} l^{-}$in the Standard Model with Reduced Theoretical Uncertainty, Phys. Rev. Lett. 112 (2014) 101801 [arXiv:1311.0903] [InSPIRE].

[11] Fermilab Lattice and MiLC collaborations, A. Bazavov et al., $B_{(s)}^{0}$-mixing matrix elements from lattice QCD for the Standard Model and beyond, Phys. Rev. D 93 (2016) 113016 [arXiv: 1602.03560] [INSPIRE].

[12] I. Dunietz, R. Fleischer and U. Nierste, In pursuit of new physics with $B_{s}$ decays, Phys. Rev. D 63 (2001) 114015 [hep-ph/0012219] [INSPIRE].

[13] K. De Bruyn, R. Fleischer, R. Knegjens, P. Koppenburg, M. Merk and N. Tuning, Branching Ratio Measurements of $B_{s}$ Decays, Phys. Rev. D 86 (2012) 014027 [arXiv:1204.1735] [INSPIRE].

[14] K. De Bruyn, R. Fleischer, R. Knegjens, P. Koppenburg, M. Merk, A. Pellegrino et al., Probing New Physics via the $B_{s}^{0} \rightarrow \mu^{+} \mu^{-}$Effective Lifetime, Phys. Rev. Lett. 109 (2012) 041801 [arXiv: 1204.1737] [INSPIRE].

[15] S. Aoki et al., Review of lattice results concerning low-energy particle physics, Eur. Phys. J. C 77 (2017) 112 [arXiv: 1607.00299] [INSPIRE].

[16] SWME collaboration, B.J. Choi et al., Kaon BSM B-parameters using improved staggered fermions from $N_{f}=2+1$ unquenched QCD, Phys. Rev. D 93 (2016) 014511 [arXiv: 1509.00592] [INSPIRE]. 
[17] N. Cho, X.-Q. Li, F. Su and X. Zhang, $K^{0}-\bar{K}^{0}$ Mixing in the Minimal Flavor-Violating Two-Higgs-Doublet Models, Adv. High Energy Phys. 2017 (2017) 2863647.

[18] L. Di Luzio, M. Kirk and A. Lenz, Updated $B_{s}$-mixing constraints on new physics models for $b \rightarrow s \ell^{+} \ell^{-}$anomalies, Phys. Rev. D 97 (2018) 095035 [arXiv:1712.06572] [InSPIRE].

[19] Particle Data Group collaboration, C. Patrignani et al., Review of Particle Physics, Chin. Phys. C 40 (2016) 100001 [inSPIRE].

[20] HFLAV collaboration, Y. Amhis et al., Averages of b-hadron, c-hadron and $\tau$-lepton properties as of summer 2016, Eur. Phys. J. C 77 (2017) 895 [arXiv:1612.07233] [INSPIRE].

[21] M. Artuso, G. Borissov and A. Lenz, CP violation in the $B_{s}^{0}$ system, Rev. Mod. Phys. $8 \mathbf{8}$ (2016) 045002.

[22] A.J. Buras, Weak Hamiltonian, CP-violation and rare decays, in Probing the standard model of particle interactions. Proceedings, Summer School in Theoretical Physics, NATO Advanced Study Institute, 68th session, Les Houches, France, July 28-September 5, 1997. Pt. 1, 2, pp. 281-539 (1998) [hep-ph/9806471] [INSPIRE].

[23] A.J. Buras, S. Jager and J. Urban, Master formulae for Delta F=2 NLO QCD factors in the standard model and beyond, Nucl. Phys. B 605 (2001) 600 [hep-ph/0102316] [INSPIRE].

[24] C.-W. Chiang, X.-G. He, F. Ye and X.-B. Yuan, Constraints and Implications on Higgs FCNC Couplings from Precision Measurement of $B_{s} \rightarrow \mu^{+} \mu^{-}$Decay, Phys. Rev. D 96 (2017) 035032 [arXiv: 1703.06289] [INSPIRE].

[25] X.-Q. Li, J. Lu and A. Pich, $B_{s, d}^{0} \rightarrow \ell^{+} \ell^{-}$Decays in the Aligned Two-Higgs-Doublet Model, JHEP 06 (2014) 022 [arXiv: 1404.5865] [INSPIRE].

[26] T. Inami and C.S. Lim, Effects of Superheavy Quarks and Leptons in Low-Energy Weak Processes $K_{L} \rightarrow \mu \bar{\mu}, K^{+} \rightarrow \pi^{+} \nu \bar{\nu}$ and $K^{0} \rightarrow \bar{K}^{0}$, Prog. Theor. Phys. 65 (1981) 297 [Erratum ibid. 65 (1981) 1772] [INSPIRE].

[27] J.A. Bagger, K.T. Matchev and R.-J. Zhang, QCD corrections to flavor changing neutral currents in the supersymmetric standard model, Phys. Lett. B 412 (1997) 77 [hep-ph/9707225] [INSPIRE].

[28] M. Blanke, A.J. Buras, K. Gemmler and T. Heidsieck, $\Delta F=2$ observables and $B \rightarrow X_{q} \gamma$ decays in the Left-Right Model: Higgs particles striking back, JHEP 03 (2012) 024 [arXiv:1111.5014] [INSPIRE].

[29] A.J. Buras, M. Misiak and J. Urban, Two loop QCD anomalous dimensions of flavor changing four quark operators within and beyond the standard model, Nucl. Phys. B 586 (2000) 397 [hep-ph/0005183] [INSPIRE].

[30] M. Gorbahn, S. Jager, U. Nierste and S. Trine, The supersymmetric Higgs sector and $B-\bar{B}$ mixing for large tan $\beta$, Phys. Rev. D 84 (2011) 034030 [arXiv:0901.2065] [InSPIRE].

[31] F. Gabbiani, E. Gabrielli, A. Masiero and L. Silvestrini, A Complete analysis of FCNC and CP constraints in general SUSY extensions of the standard model, Nucl. Phys. B 477 (1996) 321 [hep-ph/9604387] [INSPIRE].

[32] E. Golowich, J. Hewett, S. Pakvasa and A.A. Petrov, Implications of $D^{0}-\bar{D}^{0}$ Mixing for New Physics, Phys. Rev. D 76 (2007) 095009 [arXiv:0705.3650] [INSPIRE].

[33] A.J. Buras, R. Fleischer, J. Girrbach and R. Knegjens, Probing New Physics with the $B_{s} \rightarrow \mu^{+} \mu^{-}$Time-Dependent Rate, JHEP 07 (2013) 77 [arXiv:1303.3820] [INSPIRE]. 
[34] R. Fleischer, D.G. Espinosa, R. Jaarsma and G. Tetlalmatzi-Xolocotzi, CP Violation in Leptonic Rare $B_{s}^{0}$ Decays as a Probe of New Physics, Eur. Phys. J. C 78 (2018) 1 [arXiv: 1709.04735] [INSPIRE].

[35] J. Charles et al., Current status of the Standard Model CKM fit and constraints on $\Delta F=2$ New Physics, Phys. Rev. D 91 (2015) 073007 [arXiv: 1501.05013] [InSPIRE].

[36] W. Altmannshofer, S. Gori, D.J. Robinson and D. Tuckler, The Flavor-locked Flavorful Two Higgs Doublet Model, JHEP 03 (2018) 129 [arXiv:1712.01847] [INSPIRE].

[37] G. Buchalla, A.J. Buras and M.E. Lautenbacher, Weak decays beyond leading logarithms, Rev. Mod. Phys. 68 (1996) 1125 [hep-ph/9512380] [INSPIRE].

[38] G. Buchalla and A.J. Buras, QCD corrections to rare $K$ and $B$ decays for arbitrary top quark mass, Nucl. Phys. B 400 (1993) 225 [INSPIRE].

[39] M. Misiak and J. Urban, QCD corrections to FCNC decays mediated by $Z$ penguins and $W$ boxes, Phys. Lett. B 451 (1999) 161 [hep-ph/9901278] [INSPIRE].

[40] G. Buchalla and A.J. Buras, The rare decays $K \rightarrow \pi \nu \bar{\nu}, B \rightarrow X \nu \bar{\nu}$ and $B \rightarrow l^{+} l^{-}: A n$ Update, Nucl. Phys. B 548 (1999) 309 [hep-ph/9901288] [INSPIRE].

[41] C. Bobeth, M. Gorbahn and E. Stamou, Electroweak Corrections to $B_{s, d} \rightarrow \ell^{+} \ell^{-}$, Phys. Rev. D 89 (2014) 034023 [arXiv:1311.1348] [INSPIRE].

[42] T. Hermann, M. Misiak and M. Steinhauser, Three-loop QCD corrections to $B_{s} \rightarrow \mu^{+} \mu^{-}$, JHEP 12 (2013) 097 [arXiv: 1311.1347] [INSPIRE].

[43] I. Dunietz and J.L. Rosner, Time Dependent CP-violation Effects in $B^{0}-\overline{B^{0}}$ Systems, Phys. Rev. D 34 (1986) 1404 [INSPIRE].

[44] Z.-z. Xing, H. Zhang and S. Zhou, Impacts of the Higgs mass on vacuum stability, running fermion masses and two-body Higgs decays, Phys. Rev. D 86 (2012) 013013 [arXiv:1112.3112] [INSPIRE].

[45] C. Bobeth, A.J. Buras, A. Celis and M. Jung, Patterns of Flavour Violation in Models with Vector-Like Quarks, JHEP 04 (2017) 079 [arXiv: 1609.04783] [INSPIRE].

[46] A.J. Buras, D. Guadagnoli and G. Isidori, On $\epsilon_{K}$ Beyond Lowest Order in the Operator Product Expansion, Phys. Lett. B 688 (2010) 309 [arXiv:1002.3612] [INSPIRE].

[47] J.-M. Gerard, C. Smith and S. Trine, Radiative kaon decays and the penguin contribution to the Delta $I=1 / 2$ rule, Nucl. Phys. B 730 (2005) 1 [hep-ph/0508189] [INSPIRE].

[48] A. Bazavov et al., Short-distance matrix elements for $D^{0}$-meson mixing for $N_{f}=2+1$ lattice QCD, Phys. Rev. D 97 (2018) 034513 [arXiv:1706.04622] [INSPIRE].

[49] M. Blanke and A. Crivellin, B Meson Anomalies in a Pati-Salam Model within the Randall-Sundrum Background, Phys. Rev. Lett. 121 (2018) 011801 [arXiv:1801.07256] [INSPIRE].

[50] Y. Li and C.-D. Lü, Recent Anomalies in B Physics, Sci. Bull. 63 (2018) 267 [arXiv: 1808.02990] [INSPIRE]. 